\title{
Formulation and in vitro characterization of rifampicin-loaded porous poly ( $\varepsilon$-caprolactone) microspheres for sustained skeletal delivery
}

This article was published in the following Dove Press journal: Drug Design, Development and Therapy

\author{
Quanjing $M \mathrm{i}^{1}{ }^{1, *}$ \\ Peipei Luol,* \\ Yi Zuo' \\ Jidong $\mathrm{Li}^{1}$ \\ Qin Zou' \\ Yubao Li' \\ Dianming Jiang ${ }^{2}$ \\ Yaning Wang ${ }^{3}$
}

\begin{abstract}
'Research Center for Nano Biomaterials, Analytical and Testing Center, Sichuan University, Chengdu, People's Republic of China; ${ }^{2}$ Center of Bone and Trauma, The Third Affiliated Hospital of Chongqing Medical University, Chongqing, People's Republic of China; ${ }^{3}$ National Engineering Research Center for Biomaterials, Sichuan University, Chengdu, People's Republic of China

*These authors contributed equally to this work
\end{abstract}

Purpose: Mycobacterium tuberculosis is a serious public health problem affecting hundreds of millions of elderly people worldwide, which is difficult to be treated by traditional methods because of the peculiarity of skeletal system and liver damage caused by high-dose administration. In this research, a porous drug release system has been attempted to encapsulate rifampicin (RIF) into poly ( $\varepsilon$-caprolactone) (PCL) microspheres to improve the efficacy and benefit of anti-tuberculosis drug in skeletal system.

Materials and methods: The microspheres prepared by two different methods, oil-in-oil (o/o) emulsion solvent evaporation method and oil-in-water $(\mathrm{o} / \mathrm{w})$ method, were characterized in terms of morphology, size, encapsulation efficiency, drug distribution, degradation, and crystallinity. Results: The microspheres exhibited a porous structure with evenly drug distribution prepared by o/o emulsion solvent evaporation method, and their diameter ranged from 50.54 to $57.34 \mu \mathrm{m}$. The encapsulation efficiency was up to $61.86 \%$ when drug-loading content was only $1.51 \%$, and showed a little decrease with the drug-loading content increasing. In vitro release studies revealed that the drug release from porous microspheres was controlled by non-Fickian diffusion, and almost $80 \%$ of the RIF were completely released after 10 days. The results of RIF-loaded microspheres on the antibacterial activity against Staphylococcus aureus proved that the porous microspheres had strong antibacterial ability. In addition, the polymer crystallinity had prominent influence on the degradation rate of microspheres regardless of the morphology.

Conclusion: It was an efficient way to entrap slightly water-soluble drug like RIF into PCL by o/o emulsion solvent evaporation method with uniform drug distribution. The RIF-loaded porous PCL microspheres showed the combination of good antimicrobial properties and excellent cytocompatibility, and it could generate gentle environment by PCL slow degradation.

Keywords: rifampicin-loaded porous microspheres, poly ( $\varepsilon$-caprolactone), oil-in-oil solvent evaporation method, encapsulation efficiency, antibacterial ability

\section{Introduction}

Mycobacterium tuberculosis (MTB) is a serious public health problem affecting hundreds of millions of elderly people worldwide, with nearly one-third of the global population infected by MTB and latent MTB infection. ${ }^{1,2}$ It is worth noting that the skeletal system was involved in 1\%-3\% of tuberculosis (TB) cases, and extraspinal TB could cause osteomyelitis or arthritis in any peripheral bones or joints. ${ }^{3,4}$ Currently, surgical intervention has been developed as a general rule for osteoarticular TB treatment. ${ }^{4}$ According to the World Health Organization guidelines, this recommended treatment needs a long-term preoperative and postoperative drug therapy, which is a minimum of 8 months for the injectable agent and 20 months for total treatment time. ${ }^{5}$ Due to 
the peculiarity of skeletal system, deep position in the body, the efficacy of systemic administration-traditional delivery methods is not successful for deep bacilli. The treatment of MTB even to multidrug-resistant tuberculosis (MDR-TB) is a major challenge because of serious side effects, such as liver damage caused by high-dose administration. ${ }^{6}$

Drug release delivery system using biodegradable polymeric materials has been identified as an effective method to optimize traditional drug dosage, which delivers medicine to the desired position in a controlled and prolonged way, and which avoids potentially systemic toxicity by high concentration. ${ }^{7-9}$ Several studies have attempted to fabricate the delivery system using polyesters such as poly(lactideco-glycolide) (PLGA) and polylactic acid (PLA) for the treatment of pulmonary TB. ${ }^{10,11}$ However, specific adverse tissue response in the patients has been characterized as an inflammatory foreign body reaction; these patients were operated on using fixation devices made of polyglycolic acid or PLA. ${ }^{12,13}$ A painful erythematous papule and extensive osteolytic lesions developed at the implant tracks in a few weeks till years after operation. Apparently, PLGA and PLA are not the promising materials for the sustained skeletal delivery.

Different from these polyesters, poly ( $\varepsilon$-caprolactone) (PCL) has been investigated widely as the carriers for drug because of its biocompatibility, biodegradability, and high permeability to micromolecular drugs. ${ }^{10,14,15}$ Moreover, the mild and long-term degradability of PCL makes it an appropriate vehicle for antitubercular drug delivery with nonspecific tissue inflammatory response, which is different from the autocatalytic degradable polyesters such as PLGA and PLA. ${ }^{16,17}$ Rifampicin (RIF) was selected as a pharmaceutical model based on the fact that it belongs to the four first-line drugs in TB chemotherapy and the most widely investigated. ${ }^{18,19}$ Especially, RIF has not only a broad antibacterial spectrum for gram-positive and -negative bacteria and protozoa, but also antibacterial activity for MDR-TB. ${ }^{1}$ In contrast to other antibiotics, RIF binds directly to an enzyme involved in RNA polymerization, namely DNAdependent RNA polymerase; the same enzyme, present in a variety of eukaryotic species, is completely resistant to the actions of RIF. ${ }^{11}$

Drug-loaded microspheres (MS) have received extensive attention for the treatment of tuberculous osteomyelitis as they can penetrate well into bone tissue and have been extensively used for the treatment of other types of bacterial osteomyelitis, that is, osteoarthritis, spondylitis, spondylodisciitis, and arthritis. ${ }^{20,21}$ A number of methods adopted to fabricate the PCL MS are described in detail by Dash and Konkimalla. ${ }^{22}$ Among them, the emulsion solvent evaporation techniques such as oil-in-water $(\mathrm{o} / \mathrm{w}),{ }^{23}$ oil-in-oil $(\mathrm{o} / \mathrm{o}),{ }^{24}$ water-in-oil-in-water $(\mathrm{w} / \mathrm{o} / \mathrm{w}),{ }^{25}$ and soil-in-oil-inwater ${ }^{26}$ methods are widely applied to prepare polymer MS. However, the choice of an appropriate method will give rise to an efficient drug encapsulation and desired drug release behavior. Therefore, we adapted an o/o system to improve the entrapment efficiency of RIF, compared to o/w and w/o/w systems that the hydrophobic drug can easily run off. ${ }^{27,28} \mathrm{On}$ the other hand, MS fabricated by different solvent evaporation methods would also exhibit a broad range of morphologies such as being nonporous, microporous, macroporous, asymmetrically porous, and hollow depending on the rate of solvent removal and inner phase property, as well as features of continuous phase. ${ }^{26}$ For now, seldom, studies discussed the relationship between porous structure and matrix degradation behavior of PCL MS.

In our earlier work, we fabricated porous PCL MS by o/o method, which significantly improved the water-solubility drug entrapment efficiency. ${ }^{29}$ Based on the previous study, the objective of present research was to prepare hydrophobic RIF-loaded porous PCL MS with sufficient drugloading ratio, as well as controlled drug release behavior and antibacterial activity. We also investigated the effect of porous morphology on enzymatic degradation behavior of MS combined with their crystalline properties. Therefore, porous PCL MS with different drug-loading content were fabricated by o/o emulsion solvent evaporation method, and the morphology, drug distribution, particle size, encapsulation efficiency, in vitro release behavior, and antibacterial efficacy were well studied. The dense MS (DMS) prepared by o/w method were also used as controls.

\section{Materials and methods \\ Materials}

PCL (molecular weight [MW] 35,000) was purchased from Bright China Industrial Co. (Shenzhen, People's Republic of China). RIF supplied by Sigma Aldrich, Co. (St Louis, MO, USA) was used as a model drug. Pseudomonas cepacia lipase used for accelerating the cleavage rate of fatty acid esters was purchased from Sigma Aldrich, Co. Acetone, liquid paraffin, SPAN80, dichloroethane (DCE), polyvinyl alcohol, n-hexane, and methanol were obtained from Changzheng Huabo, Ltd. (Chengdu, People's Republic of China), and all chemical reagents were of analytical reagent level. 


\section{Method fabrication}

The preparation of MS was based on o/o emulsificationsolvent evaporation methods. We also prepared MS by $\mathrm{o} / \mathrm{w}$ emulsification-solvent evaporation methods for comparison.

For the o/o method, the following conditions were used according to the formulae listed in Table 1: PCL $(1 \mathrm{~g})$ was dissolved in $10 \mathrm{~mL}$ of acetone, which contained different concentrations of RIF, then dropped into liquid paraffin containing surfactant SPAN80 at room temperature using agitator at 1,500 rpm for $15 \mathrm{~min}$. Subsequently, the resulting emulsion was stirred at $500 \mathrm{rpm}$ for $1 \mathrm{~h}$ to allow the acetone evaporation and MS precipitation. The MS were centrifuged at 2,500 rpm for $5 \mathrm{~min}$, washed with n-hexane, and dried at room temperature in the vacuum. Blank MS were obtained by the same method for the degradation experiments.

For the o/w method, $1 \mathrm{~g}$ of PCL and $50 \mathrm{mg}$ RIF were dispersed in a DCE $(10 \mathrm{~mL})$, and dropped into $100 \mathrm{~mL}$ water containing $2 \%$ polyvinyl alcohol $(\mathrm{w} / \mathrm{v})$. An emulsion was formed by stirring for $30 \mathrm{~min}$, subsequently residual solvent was evaporated for $1.5 \mathrm{~h}$, and the MS were recovered by centrifugation and dried.

\section{MS characterization}

The internal and external morphology of MS and the surface structures were observed by scanning electron microscopy (SEM, 6500LV; JEOL, Tokyo, Japan). MS were coated with gold under vacuum before SEM. The particle size was measured for $>400$ particles by imaging software (ImageJ).
The distribution of RIF in MS was observed by optical microscope (Nikon TE2000-U), and the pictures were taken at $10 \mathrm{~min}$ after emulsion droplets formed. As a comparison, the drug-free MS were also observed at the same step.

\section{Encapsulation efficacy}

RIF-loaded MS (10 mg) were immersed into $10 \mathrm{~mL}$ methanol for $24 \mathrm{~h}$ in a shaker. The drug extracted from the resulting solution was measured at the maximum wavelength of $334 \mathrm{~nm}$ using a UV-Vis spectrophotometer (SP-754; Shanghai Spectrum Co., Shanghai, People's Republic of China), and the concentration $\left(W_{\mathrm{d}}\right)$ of the drug entrapped in MS was calculated by a calibration curve. Each group of procedures was carried out in triplicate. The percentages of drug-loading ratio and entrapment efficiency were calculated by Equations 1-3:

$$
\text { Drug-loading ratio }(\%)=\frac{W_{\mathrm{d}}}{W_{\mathrm{m}}} \times 100 \%
$$

Theoretical drug-loading ratio $(\%)=\frac{W_{\mathrm{d}}^{\prime}}{W_{\mathrm{d}}^{\prime}+W_{\mathrm{m}}^{\prime}} \times 100 \%$

Entrapment efficiency (\%)

$$
=\frac{\text { Drug-loading ratio }}{\text { Theoretical drug-loading ratio }} \times 100 \%
$$

where $W_{\mathrm{d}}$ is the calculated weight of RIF in drug-loaded MS (g), $W_{\mathrm{m}}$ is the weight of drug-loaded miscrospheres (g),

Table I Preparation and characterization of PCL microspheres prepared from different formulae using o/o and o/w methods

\begin{tabular}{|c|c|c|c|c|c|c|c|c|}
\hline Samples & $\begin{array}{l}\text { o/o } \\
\text { acetone:petrol } \\
(w / w)\end{array}$ & $\begin{array}{l}\text { SPAN80 } \\
(\%, w / v)\end{array}$ & $\begin{array}{l}\text { Drug dosages } \\
\text { ratio PCL:Drug } \\
(w / w)\end{array}$ & $\begin{array}{l}\text { Theoretical } \\
\text { drug loading } \\
\text { (\%) }\end{array}$ & $\begin{array}{l}\text { Drug } \\
\text { loading (\%) }\end{array}$ & $\begin{array}{l}\text { Encapsulation } \\
\text { efficiency (\%) }\end{array}$ & $\begin{array}{l}\text { Mean } \\
\text { diameter } \\
(\mu \mathrm{m})\end{array}$ & $\begin{array}{l}D_{10}-D_{90} \\
(\mu \mathrm{m})\end{array}$ \\
\hline RIF-loaded & $1: 3$ & 3 & $20: 1$ & 4.76 & $2.64 \pm 0.16$ & $55.54 \pm 3.39$ & 50.56 & $29.97-72.78$ \\
\hline porous & I:5 & 3 & $20: 1$ & 4.76 & $2.57 \pm 0.29$ & $53.99 \pm 6.01$ & 59.46 & $28.42-99.58$ \\
\hline \multirow[t]{9}{*}{ microspheres } & $\mathrm{I}: 3$ & I & $20: 1$ & 4.76 & $2.07 \pm 0.12$ & $43.49 \pm 2.55$ & 56.64 & $28.54-87.14$ \\
\hline & $1: 3$ & 2 & $20: 1$ & 4.76 & $2.49 \pm 0.08$ & $52.38 \pm 1.79$ & 54.25 & $28.63-87.46$ \\
\hline & $1: 3$ & 3 & $20: 1$ & 4.76 & $2.64 \pm 0.16$ & $55.54 \pm 3.39$ & 50.56 & $29.97-72.78$ \\
\hline & $1: 3$ & 3 & $20: 0$ & 0 & 0 & 0 & 52.35 & $24.66-81.98$ \\
\hline & $\mathrm{I}: 3$ & 3 & $20: 0.5$ & 2.44 & $\mathrm{I} .5 \mathrm{I} \pm 0.04$ & $61.86 \pm 1.70$ & 50.54 & $22.11-85.49$ \\
\hline & $1: 3$ & 3 & $20: 1$ & 4.76 & $2.64 \pm 0.16$ & $55.54 \pm 3.39$ & 50.56 & $29.97-72.78$ \\
\hline & $1: 3$ & 3 & $20: 1.5$ & 6.98 & $4.05 \pm 0.14$ & $57.96 \pm 2.01$ & 52.30 & $20.14-89.73$ \\
\hline & $1: 3$ & 3 & $20: 2$ & 9.09 & $5.16 \pm 0.74$ & $56.76 \pm 8.10$ & 57.34 & $31.82-86.53$ \\
\hline & $\begin{array}{l}\text { o/w, DCE: } 2 \% \\
\text { PVA water }\end{array}$ & & $\begin{array}{l}\text { Drug dosages ratio } \\
\text { PCL:drug }(w / w)\end{array}$ & $\begin{array}{l}\text { Theoretical } \\
\text { drug loading (\%) }\end{array}$ & $\begin{array}{l}\text { Drug loading } \\
\text { (\%) }\end{array}$ & $\begin{array}{l}\text { Encapsulation } \\
\text { efficiency (\%) }\end{array}$ & $\begin{array}{l}\text { Mean diameter } \\
(\mu \mathrm{m})\end{array}$ & $\mathrm{D}_{10}-\mathrm{D}_{90}(\mu \mathrm{m})$ \\
\hline RIF-loaded & $\mathrm{I}: 10$ & - & $20: 0$ & 0 & 0 & 0 & $20.6 \mathrm{I}$ & $9.68-34.33$ \\
\hline dense & $\mathrm{I}: 10$ & - & $20: 1$ & 4.76 & $0.58 \pm 0.13$ & $12.27 \pm 2.34$ & 27.49 & $2.33-40.99$ \\
\hline
\end{tabular}
(mean \pm SD, $n=3$ )

Abbreviations: DCE, dichloroethane; o/o, oil-in-oil; o/w, oil-in-water; PCL, poly ( $\varepsilon$-caprolactone); PVA, polyvinyl alcohol; RIF, rifampicin. 
$W_{\mathrm{d}}^{\prime}$ is the total amount of RIF used for particle preparation $(\mathrm{g})$, and $W_{\mathrm{m}}^{\prime}$ is the total weight of PCL used for preparation $(\mathrm{g})$.

\section{In vitro release study}

The release study was carried out in triplicate. Briefly, the dialysis bags (exclusion size $1.4 \times 10^{4} \mathrm{MW}$ ) with $20 \mathrm{mg}$ RIF-loaded PCL MS were placed into a glass tube containing $5 \mathrm{~mL}$ PBS $(0.2 \mathrm{M}, \mathrm{pH}=7.4)$ and incubated at $37^{\circ} \mathrm{C}$ in a shaker. At specified time intervals, the release medium was completely removed and replaced with $5 \mathrm{~mL}$ fresh PBS. RIF concentration was measured by the absorbance of elution medium at $332 \mathrm{~nm}$ using a UV-Vis spectrophotometer. The results were plotted in terms of cumulative releases (calculated by Equation 4).

$$
\text { Cumulative amount released }(\%)=\left(\frac{M_{\mathrm{t}}}{M_{\infty}}\right) \times 100
$$

where $M_{\mathrm{t}}$ and $M_{\infty}$ are the cumulative amount of drug release at time $t$ and infinite time from matrix, respectively.

Equation 5 was utilized for the mathematics characterization of the release kinetics of RIF from the PCL MS.

$$
\frac{M_{\mathrm{t}}}{M_{\infty}}=k t^{n}\left(\frac{M_{\mathrm{t}}}{M_{\infty}} \leq 0.6\right)
$$

where $k$ is a constant incorporating characterization of the matrix and the drug, and $n$ is the release exponent, which is indicative of the transport mechanism. ${ }^{30}$

\section{Antibacterial activity assay}

The antibacterial activity of the RIF-loaded MS was examined against Staphylococcus aureus. ${ }^{31}$ Specifically, bacterial spores were introduced in $5 \mathrm{~mL}$ of Mueller-Hinton broth and incubated for $12 \mathrm{~h}$. To achieve the final concentration of about $3 \times 10^{8}$ colony-forming unit (cfu) $/ \mathrm{mL}, 0.1 \mathrm{~mL}$ of the culture was diluted into another $5 \mathrm{~mL}$ of Mueller-Hinton broth. Meanwhile, molten Mueller-Hinton agar was poured into sterile dishes and allowed to solidify. Subsequently, the plates were spread with the bacterial culture, and the round filter papers with the diameter of $1 \mathrm{~cm}$ immersed into the PBScontaining drug that was released from the RIF-loaded MS were seeded in the center of plates with $100 \mu \mathrm{L} \mathrm{S}$. aureus. ${ }^{32}$ The diameter of the inhibition zone was measured and recorded. All samples were incubated at $37^{\circ} \mathrm{C}$ for $18 \mathrm{~h}$, and the results were compared with the RIF-free MS. The antibacterial activity experiments were performed in triplicate.

\section{MS degradation}

Drug-free PCL MS (10 mg), prepared by two methods mentioned above, were placed into the glass tube and $5 \mathrm{~mL}$ PBS (0.2 M, pH=7.4) with $0.2 \mathrm{mg} / \mathrm{mL}$ lipase was added..$^{33}$ The tubes were placed in a shaker at $37^{\circ} \mathrm{C}$. Degradation studies were performed with MS immersed in the medium for 2, 4, 10, 24, 48, and $72 \mathrm{~h}$. After that, the degrading medium and particles were separated at a certain time by filtration. Then the particles were washed with distilled water and dried at room temperature. The morphologic changes were observed by SEM. Meanwhile, the degradation rate of MS can be monitored by measuring the released acids from the PCL by titration. One milliliter of degrading medium collected at a specified time interval was titrated by $0.01 \mathrm{~mol} / \mathrm{L} \mathrm{NaOH}^{34}$ The consumption of $\mathrm{NaOH}$ was plotted against the time. Three samples were processed at each selected degradation time.

\section{Thermal analysis and X-ray diffraction (XRD) analysis}

The thermal characterization of drug-free PCL MS, prepared by two methods mentioned above, was measured by differential scanning calorimeter (DSC, Thermal Analyst 2000; STA449F3, Netzsch, German). Samples were heated from $0^{\circ} \mathrm{C}$ to $100^{\circ} \mathrm{C}$ with a heat increase rate of $10^{\circ} \mathrm{C} \cdot \mathrm{min}^{-1}$.

The degree of crystallinity, $X_{\mathrm{c}}(\%)$, was calculated by the following equation:

$$
X_{\mathrm{c}}(\%)=100 \times \frac{\Delta H_{\mathrm{m}}}{\Delta H_{\mathrm{F}}}
$$

where $\Delta H_{\mathrm{m}}$ and $\Delta H_{\mathrm{F}}$ were the heats of fusion of the sample and the $100 \%$ crystalline pure PCL $(136 \mathrm{~J} / \mathrm{g}),{ }^{35}$ respectively.

XRD analysis was carried out with an X-ray diffractometer (X'Pert Pro MPD; Philips, Amsterdam, Netherlands), and samples were scanned over a $2 \theta$ range of $10^{\circ}-50^{\circ}$ at the rate of $0.2^{\circ} \cdot \mathrm{min}^{-1}$.

\section{In vitro cytocompatibility evaluation}

Cellular morphology and proliferation of MG63 were used for the in vitro cytocompatibility evaluation. ${ }^{36}$ MG63 cells, purchased from the BeNa Culture Collection, Beijing, were originally derived from human osteosarcoma tissue and they expressed the characteristic features of osteoblasts. Culture of MG63 cells and in vitro cytocompatibility evaluation were processed in West China College of Stomatology, Sichuan University (Chengdu, People's Republic of China). MG63 cells were routinely cultivated in F12 medium (cell culture grade; BioWhittaker, Walkersville, MD, USA) supplemented 
with $10 \%$ volume fraction of calf serum (cell culture grade; Gibco $^{\circledR}$; Thermo Fisher Scientific, Waltham, MA, USA), 1\% penicillin/streptomycin, and $1 \% 1$-glutamine at $37^{\circ} \mathrm{C}$ under a stream of $5 \% \mathrm{CO}_{2}$.

For cell viability studies, four batches of RIF-loaded and drug-free porous and DMS were tested. Theoretical drug loading was $4.76 \%$ (w/w) for two RIF-loaded MS. Sterilized coverslips were put into 24-well plates and preincubated with $1 \mathrm{~mL}$ of culture medium overnight. Then, the medium was carefully removed, and the MS were incubated in $1 \mathrm{~mL}$ of the MG63 cell suspension (about $2 \times 10^{4}$ cells per well). The seeded specimens were cultured in a humidified incubator $\left(37^{\circ} \mathrm{C} ; 5 \% \mathrm{CO}_{2}\right)$, and the medium was changed every 3 days. After culturing for 1, 3, 5, and 7 days, cell proliferation with MS on coverslips was observed by optical microscope and then determined quantitatively using MTT assay method by measuring the activity of the mitochondrial dehydrogenases with a multilabel counter (Wallac Victor3 1420; PerkinElmer Co., Waltham, MA, USA) at $490 \mathrm{~nm} .{ }^{37}$ One of the wells without MS was set as a control group. The data from three individual experiments were averaged.

The adhesion and morphology of MG63 cells cultured with MS were also observed with SEM. The MG63 cells cultured with the specimen for 7 days were rinsed with PBS, fixed at a $2.5 \%$ volume fraction of glutaraldehyde, subjected to graded alcohol dehydration, rinsed with isoamylacetate, dried with supercritical $\mathrm{CO}_{2}$, and observed by SEM. ${ }^{38}$

\section{Statistical analysis}

Data were collected in a Microsoft Excel 2010 database and filter using Box Chart of the Origins 9.0 software. Valid data were presented as the mean \pm SD. Statistical analysis was calculated using Graphpad Instat software $\left(\operatorname{InStat}^{\circledR} 3.0\right.$; Graphpad Software Inc., La Jolla, CA, USA). The statistical analysis was carried out using one-way analysis of variance with Tukey's test for multiple comparisons, with $p<0.05$ indicating statistical significance.

\section{Results \\ Effect of formulae on PCL MS characterization}

Based on o/o emulsification-solvent evaporation method, three different batch formulas have been investigated to determine the optimum formulation of PCL MS shown in Table 1, which were the acetone-to-petrol volume ratio (o/o), surfactant value, and the RIF amounts, respectively. Meanwhile, the characterization of the size, drug-loading ratio, and entrapment efficiency of MS are given in Table 1. In first batch formula, when o/o volume ratio was 1:3, the MS were uniform and monodispersed in diameter. With the increase in petrol volume, that is, the continuous phase in the o/o method, the mean diameter and size distribution increased, while the drug-loading rate and entrapment rate had no significant difference $(p>0.05)$. In second batch formula, SPAN80 was used as the surfactant of the MS preparation, and the mean size and size distribution decreased with an increase of the surfactant content from $1 \%$ to $3 \%$. However, the amount of surfactant significantly affected the encapsulation efficiency of PCL MS $(p<0.001)$. The entrapment efficiency of RIF in MS had an incremental value of 6\%-20\% in each $1 \%$ increase in the surfactant concentration. In third batch formula, it is surprising that the drug dosage did not affect the encapsulation efficiency ratio even little when the RIF dosage ratio increased up to 20:1. The encapsulation efficiency of RIF-loaded MS had no significant difference while the theoretical drug ratios were $2.64 \%, 4.05 \%$, and $9.09 \%$, respectively $(p>0.05)$. Except for the highest drug dosage $(9.09 \%)$ of the MS, no significant difference was observed in particle sizes $(p>0.05)$.

In comparison, the PCL MS prepared by using o/w method have been studied according to the formula in Table 1. Apparently, both the drug-loading rate and encapsulation efficiency of the MS prepared by o/w method were too lower than the MS prepared in o/o system. Only $12.27 \%$ of the RIF dosage had been encapsulated in the PCL MS, which is about one-quarter of the entrapment rate of that of $\mathrm{o} / \mathrm{o}$ method. Moreover, the size of the MS from o/w system was about $50 \%$ smaller than that of o/o system.

In Figure 1, the PCL MS prepared according to the formula in Table 1 were spherical shapes. All the MS prepared by o/o method were porous structure (named as porous microspheres [PMS]), in comparison to dense particles made by o/w method (named as DMS). However, the morphology of PMS with fewer pores was observed on the surface when the drug concentration increased (Figure 1A-E). The microporous morphology was shown on the surface (Figure 1I), and heterogeneous pores with irregular size and shape were observed in the cross section of MS (Figure 1J and $\mathrm{K})$. Notably, the center feature of PMS was enlarged internal pores, whereas the shell was honeycomb-like microstructure. Besides this, abundant interpenetrated holes were observed inside MS. As the comparison, DMS presented a compact structure without any pores inside, as in Figure 1L.

As the natural color of RIF is orange, the orange color observed by optical microscope in Figure $1 \mathrm{G}$ actually reflected the distribution of RIF in MS. The optical pictures 

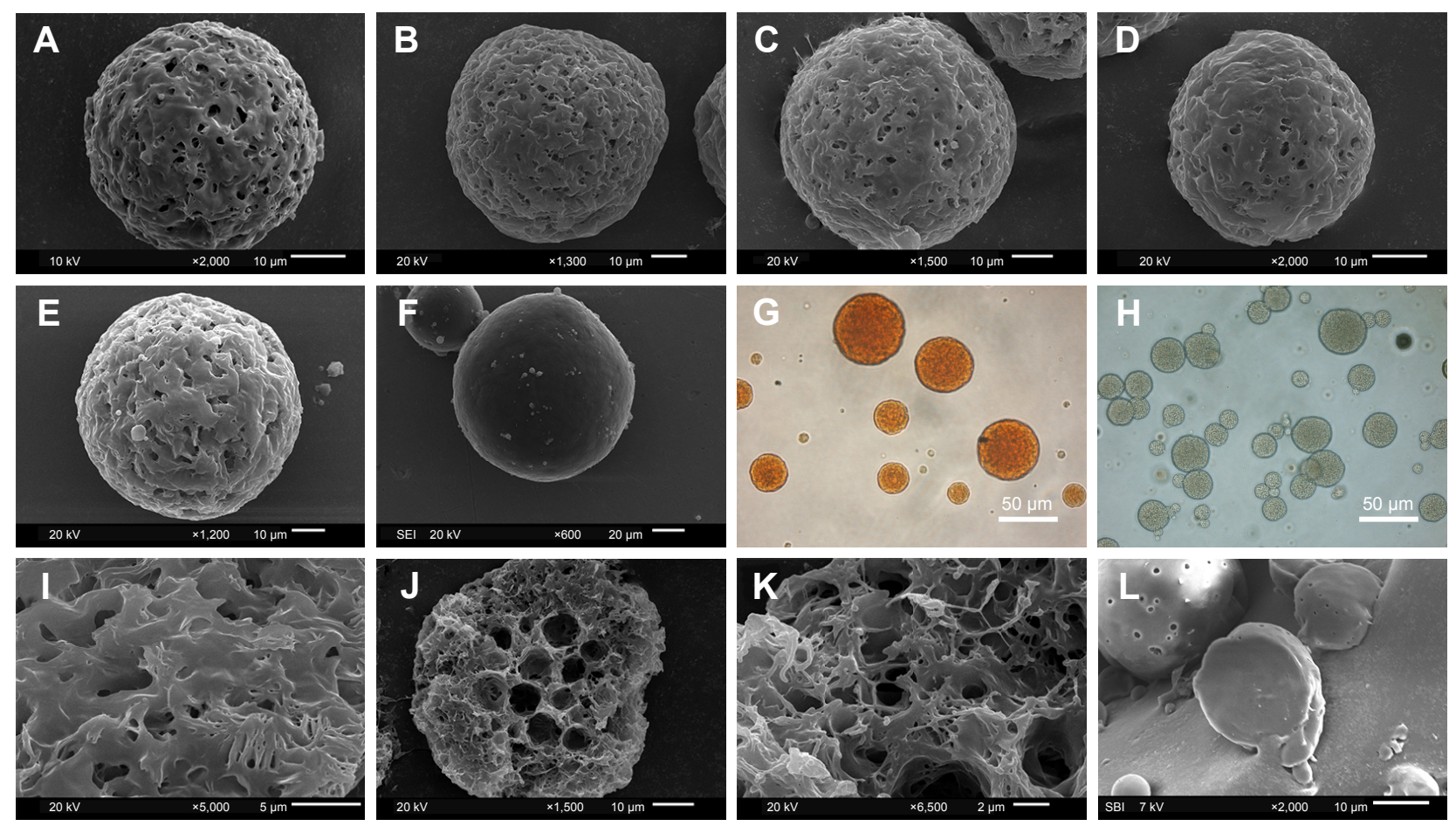

Figure I SEM pictures of RIF-loaded PCL microspheres prepared by different drug dosage ratios: (A) I.5I\% PMS; (B) 2.64\% PMS; (C) 4.05\% PMS; (D) 5.16\% PMS; (E) RIF-free PMS; and (F) RIF-free DMS. Optical pictures of microspheres before complete solidification: (G) $2.64 \%$ PMS and (H) RIF-free PMS. SEM pictures of the crosssectional view of microspheres (magnification $\times 400$ ): (I) the external morphology of RIF-free PMS; (J) the internal morphology of RIF-free PMS; (K) the magnification picture of internal morphology of RIF-free PMS; and (L) the internal morphology of RIF-free DMS.

Abbreviations: DMS, dense microspheres; PCL, poly ( $\varepsilon$-caprolactone); PMS, porous microspheres; RIF, rifampicin; SEM, scanning electron microscopy; SEI, secondary electron imaging.

showed a homogeneous distribution of RIF in porous matrix, indicating that the drug is randomly and uniformly distributed in the PCL MS. In this way, faint yellow and transparent porous MS were observed as RIF-free PMS (Figure 1H).

\section{In vitro release study and antibacterial activity assay}

In vitro drug release behaviors of porous PCL MS with various drug-loading contents were studied by comparing with pure RIF. In the case of RIF-loaded DMS obtained by o/w method, the release experiments are not available because the drug-loading content was too low $(<0.3 \%)$ to detect by UV-Vis spectrophotometer. As shown in Figure 2I, the pure RIF almost dissolved completely into PBS in $3 \mathrm{~h}$, leading to a sharp increase in drug concentration, while all RIF-loaded PMS presented a controllable drug release behavior. Specifically, the MS showed a burst release of $40 \%-50 \%$ during the first $24 \mathrm{~h}$, followed by the gradual increase in the drug amount
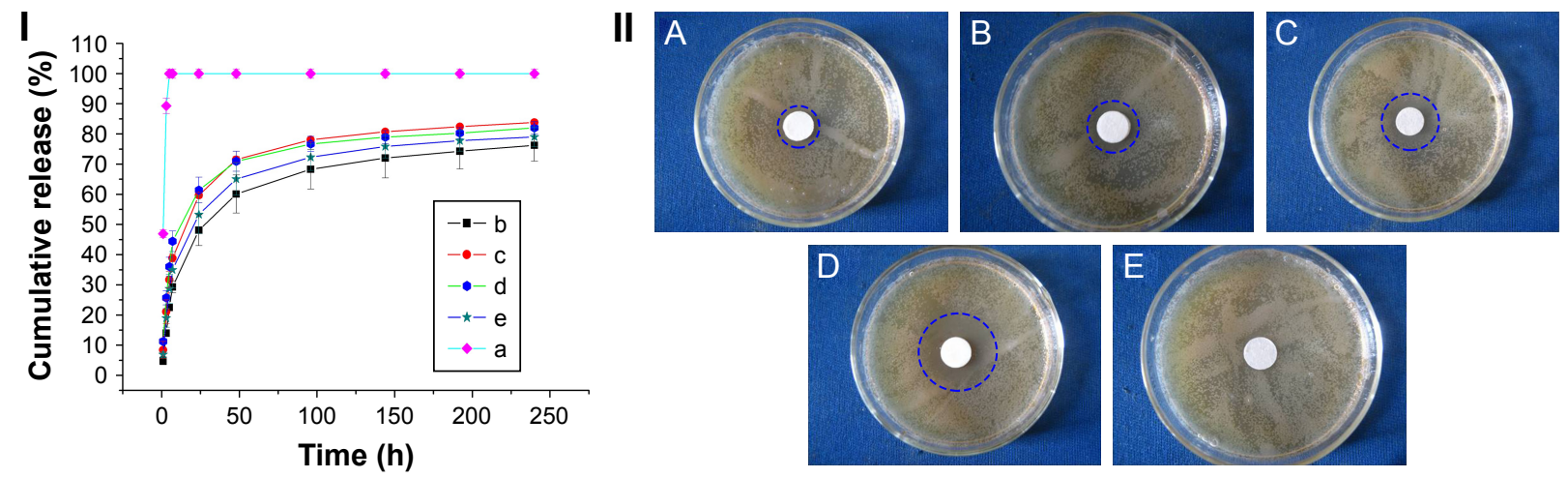

Figure 2 (I) In vitro release profile of pure RIF and drug-loaded PMS: (a) Pure RIF; (b) 1.5I\%; (c) 2.64\%; (d) 4.05\%; and (e) 5.16\%. (II) Pictures of Staphylococcus aureus incubated for $18 \mathrm{~h}$ at $37^{\circ} \mathrm{C}$ together with extracted fluid from RIF-loaded PMS: (A) I.5I\%; (B) 2.64\%; (C) 4.05\%; (D) 5.16\%; and (E) RIF-free PMS.

Abbreviations: PMS, porous microspheres; RIF, rifampicin; SEM, scanning electron microscopy. 
Table 2 Values of the release exponent $(n)$, constant $(k)$, and correlation coefficient $\left(R^{2}\right)$

\begin{tabular}{llll}
\hline PCL:RIF & $\boldsymbol{n}$ & $\boldsymbol{k}$ & $\boldsymbol{R}^{\mathbf{2}}$ \\
\hline $20: 0.5$ & 0.732 & 0.058 & 0.949 \\
$20: 1$ & 0.621 & 0.100 & 0.947 \\
$20: 1.5$ & 0.539 & 0.133 & 0.936 \\
$20: 2$ & 0.646 & 0.085 & 0.935 \\
\hline
\end{tabular}

Abbreviations: PCL, poly ( $\varepsilon$-caprolactone); RIF, rifampicin.

in sustained release. The total quantity of RIF from PCL porous MS reached almost $80 \%$ after 10 days. Moreover, the MS with the lowest drug-loading content had the lowest initial burst and the least cumulative release within 10 days, and the drug release rate increased with more drugs contained in MS. Interestingly, the MS with the highest drug-loading content did not show an anticipative highest initial burst and fastest release rate.

Table 2 listed the best-fit values for the parameters in the exponent expression of different drug release curves determined by Excel software. The data $n$ is the diffusional exponent, depending on the RIF transport mechanism and geometry of the device. The constant $k$ relates to incorporating natural characteristics of the polymer and drug, structures, and geometry characteristics of the device. The $R^{2}$ is the correlation coefficient of the experimental data associated with Equation $2 .{ }^{39}$ The results showed that all of the $n$ values were between 0.5 and 1 , and generally decreased with the rising loading level. However, the $k$ values exhibited an inverse proportion with $n$ values. In particular, the kinetic parameters of the MS that contained most of the drug were out of the tendency, and presented the intermediate values among these samples.

Figure 2II shows the images of antibacterial tests performed on solid agar plates that were covered by filter paper with or without RIF. The results showed that a clear inhibition zone was generated by inhibiting the microorganisms, while the extracted fluid acquired from RIF-free MS exhibited no inhibition ability against the proliferation of the bacteria. The diameter of inhibition zone gradually increased with the increasing concentration of RIF.

\section{In vitro degradation study}

Figure 3I shows the morphologic changes of the two types of RIF-free MS in the course of enzymatic degradation. Before degradation, the MS obtained by o/o method were porous and loose (Figure 3I and A). After degradation for $24 \mathrm{~h}$, more holes appeared on the MS surface of PMS.
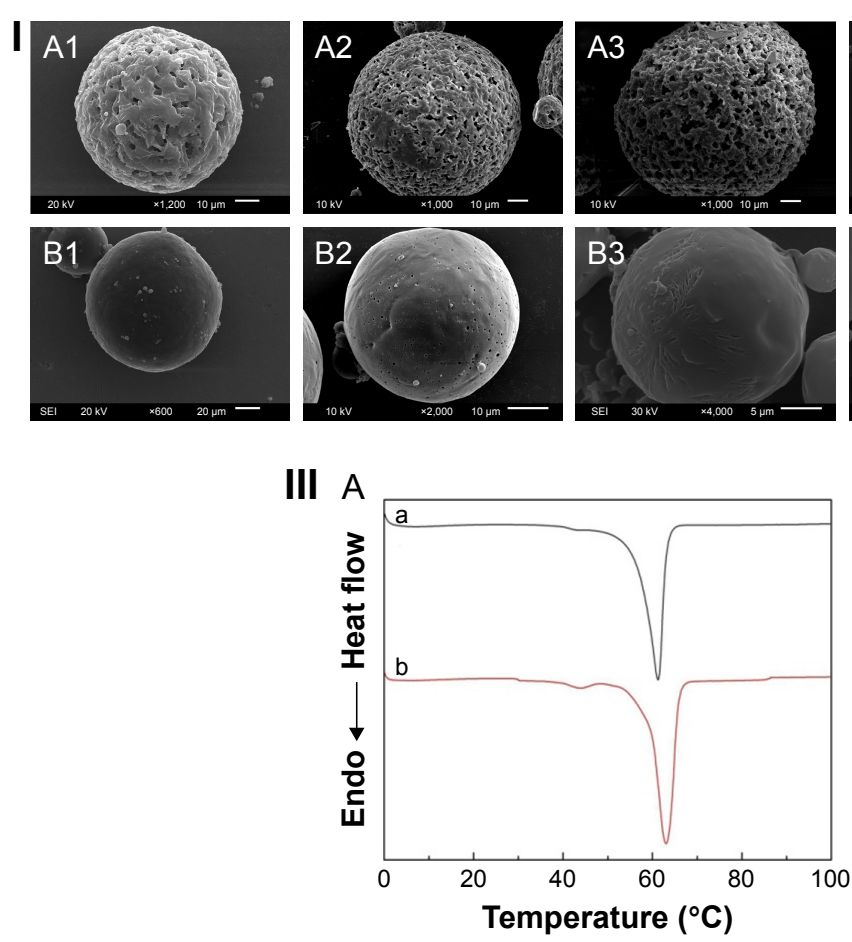
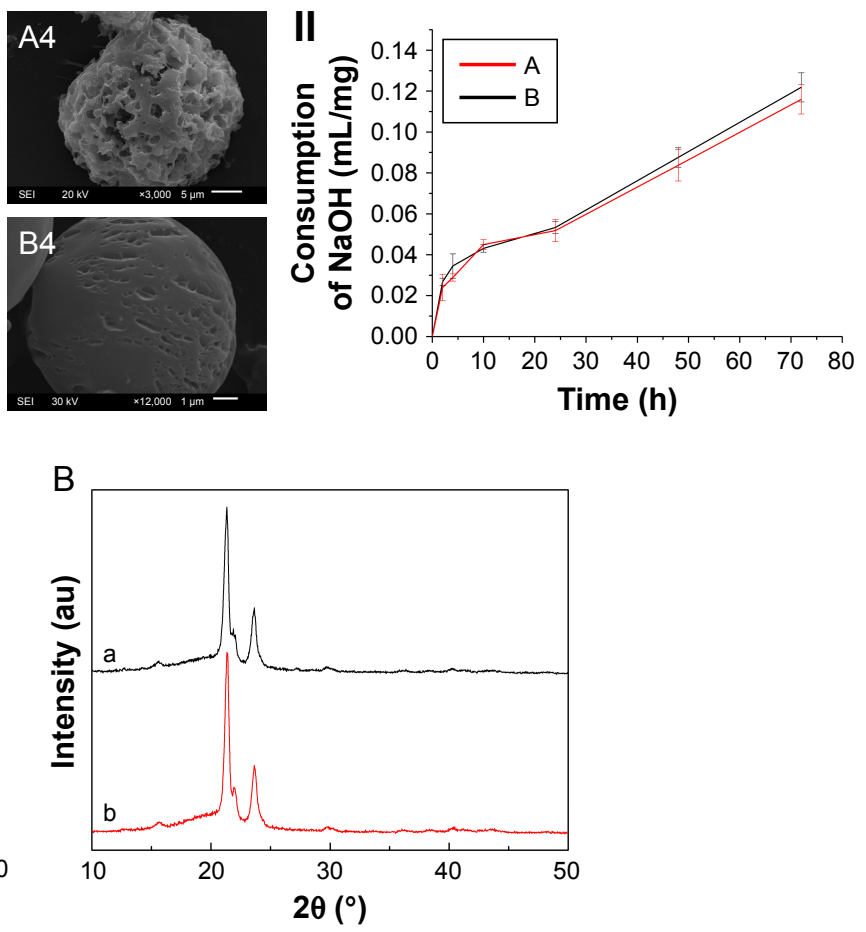

Figure 3 (I) SEM photographs of drug-free PCL microspheres during in vitro degradation prepared by oil-in-oil method ( $\mathrm{A})$ as well as oil-in-water method (B): $0 \mathrm{~h}$ ( $\mathrm{Al}$, $\mathrm{BI}$ ), $24 \mathrm{~h}$ (A2, B2), $48 \mathrm{~h}$ (A3, B3), and $72 \mathrm{~h}$ (A4, B4). (II) The consumption of $\mathrm{NaOH}$ for different samples in the course of degradation: (A) drug-free PMS; (B) drug-free DMS. (III) Analysis of the physical properties of drug-free PCL microspheres using DSC (A) and XRD (B): (a) PMS; (b) DMS.

Abbreviations: DMS, dense microspheres; DSC, differential scanning calorimeter; PCL, poly (ع-caprolactone); PMS, porous microspheres; RIF, rifampicin; SEM, scanning electron microscopy; XRD, X-ray diffraction; SEI, secondary electron imaging. 
Subsequently, the pores on the surface had enlarged and the matrix material had gradually degraded. After $72 \mathrm{~h}$, huge disruption of interconnected structured and significant erosion on surface was seen in SEM picture. However, in the case of o/w method, the morphology of MS caused by degradation was found to be significantly different on a temporal basis (Figure 3I and B). At the beginning, DMS were smooth and dense without holes and cavities. After degradation for $24 \mathrm{~h}$, some small holes were observed on the surfaces of DMS particles. With further degradation for $48 \mathrm{~h}$, the MS surfaces are generally accompanied by grooves and cracks. Thereafter, the degradation had continued and both the quantity and size of grooves and cracks had increased when the degradation time was up to $72 \mathrm{~h}$.

The titration method was utilized to directly monitor degradation rate by measuring the released carboxylic groups during the ester cleavage of PCL. Interestingly, the result indicates that there is no significant difference in degradation rate between two types of MS (Figure 3II). Previous study revealed that the degradation rate had a close connection with both the molecular weight and the degree of crystallinity. ${ }^{40}$ As both of the MS were prepared by the same molecular weight of PCL, the degree of crystallinity seemed to determine the degradation rate. To confirm this hypothesis, DSC and XRD were utilized to reflect the crystallography properties of PCL MS. (Figure 3III and A) shows the DSC thermograms of the porous and nonporous PCL MS, which displayed melting temperature at $61.2^{\circ} \mathrm{C}$ and $63.2^{\circ} \mathrm{C}$, respectively. The crystallinity corresponding to each sample was $57.53 \%$ and $61.32 \%$ through calculation. Moreover, the XRD profiles of two types of MS are shown in (Figure 3III and $B$ ). The two intense reflection peaks at $2 \theta$ values of $21.4^{\circ}$ and $23.6^{\circ}$ indexed as (110) and (200) reflections ${ }^{41}$ had been observed similarly, regardless of porous structures' presence. Herein, the results of DSC and XRD suggest that the crystallinity of the porous PCL MS is approximately equal to that of the nonporous PCL.

\section{In vitro cytocompatibility evaluation}

The viability of MG63 has been observed by using optical micrographs (Figure 4I). With the increasing incubation time, live cells multiplied gradually till day 7 , suggesting that the
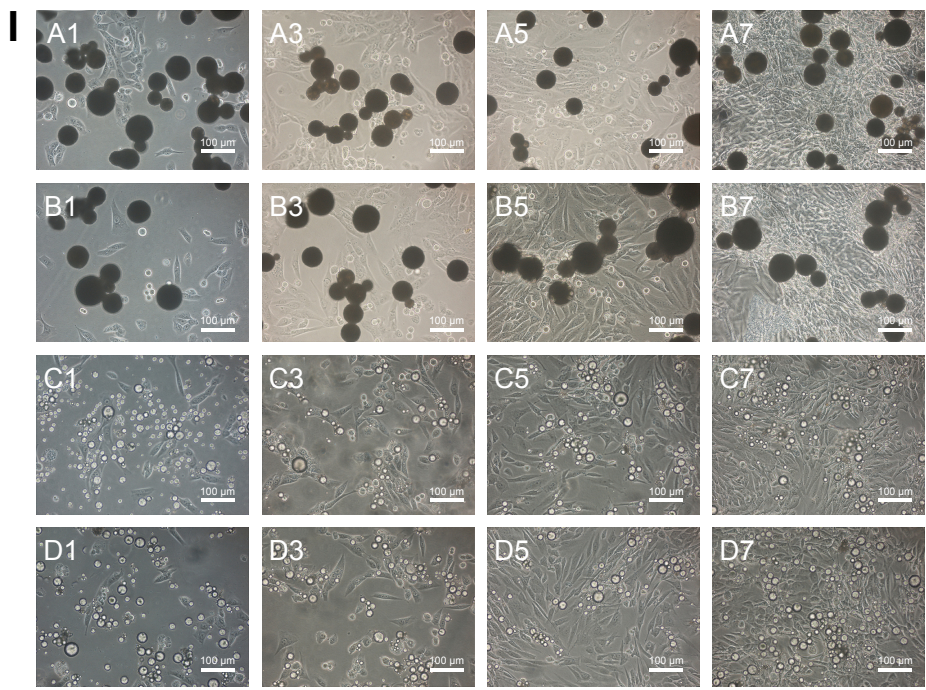

II
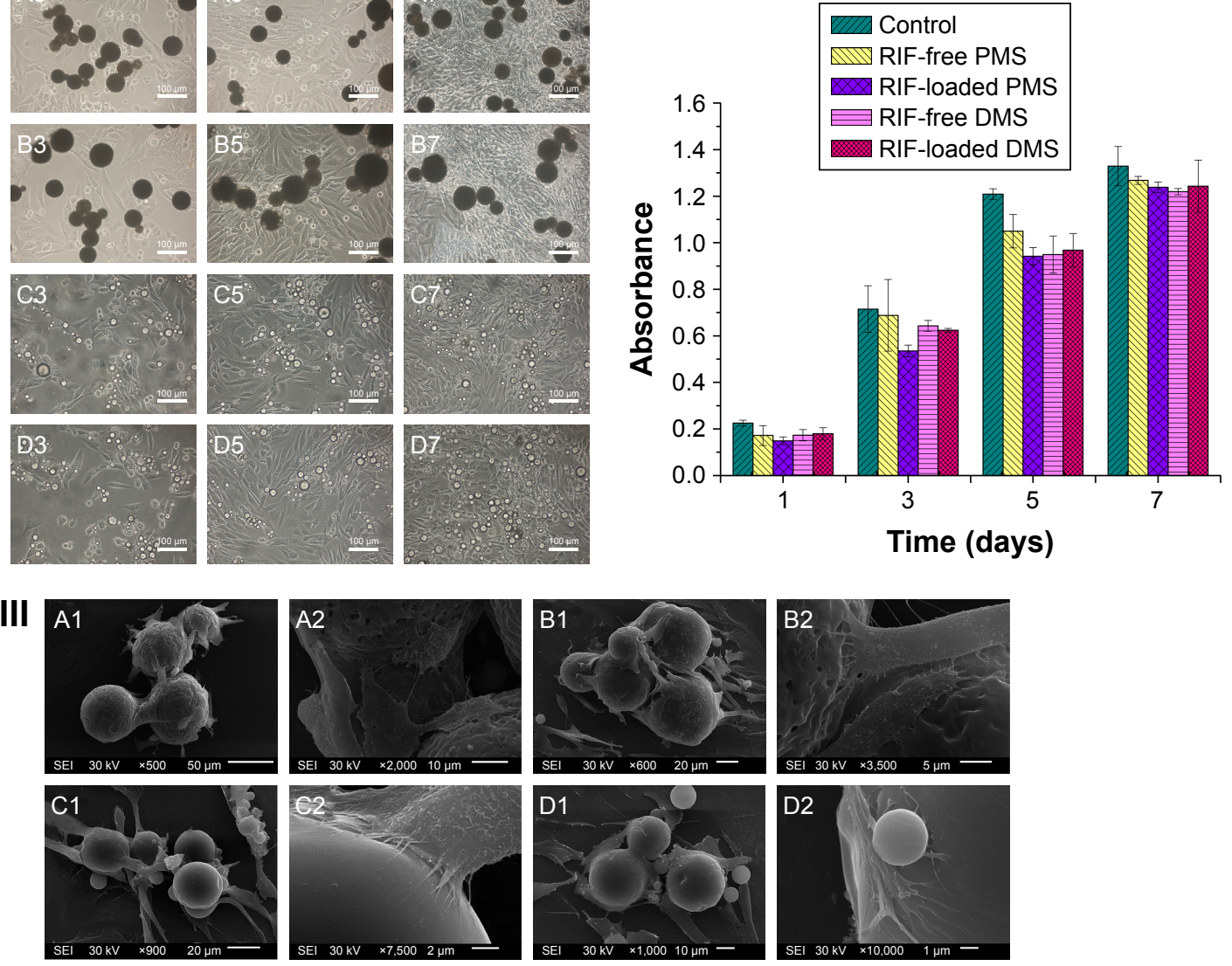

Figure 4 (I) Optical pictures of MG63 cultured with microspheres for I, 3, 5, and 7 days: (A) RIF-free PMS; (B) RIF-loaded PMS; (C) RIF-free DMS; (D) RIF-loaded DMS. (II) MTT assay of MG63 proliferation cultured with PCL microspheres (mean \pm SD, $n=3$ ). (III) SEM pictures of MG63 cells cultured with microspheres for 7 days: (A) RIF-free PMS; (B) RIF-loaded PMS; (C) RIF-free DMS; (D) RIF-loaded DMS. Magnification $\times 200$.

Abbreviations: DMS, dense microspheres; PCL, poly ( $\varepsilon$-caprolactone); PMS, porous microspheres; RIF, rifampicin; SEM, scanning electron microscopy. 
cells proliferate well on all kinds of the MS. Besides, the optical images also presented normal morphology of these osteocytes. The SEM pictures also demonstrated commendable cells attachment on the MS (Figure 4III), which is coincident with the results of optical pictures. At the same time, there were no significant differences between the porous MS and DMS on the morphology of cell, which indicated that the surface topography had a little effect on cell viability. Similar investigation has been observed by Kulkarni. ${ }^{42}$

The cell proliferation results by MTT assay are shown in Figure 4II. There was no statistically significant difference between each group on day 1 . With the increase of culture time, the proliferation value of cells showed a continued growing trend for all MS groups and the control group. However, the values of experimental groups were relatively lower than that of the negative control group. Specifically, the proliferation value of RIF-loaded PMS showed a significant difference at 3 and 5 days compared to other groups $(p<0.05)$. Because the pristine RIF has an inhibitory effect on cells, polymer-entrapped drugs could relieve the inhibition effect on cells in the initial burst stage. ${ }^{43}$ As time went on to 7 days, the inhibition effect had gradually disappeared because of the slow release of drug-loaded MS $(p>0.05)$. At the same time, the cell proliferation of porous MS had no significant difference with that of DMS $(p>0.05)$. The result indicates that the surface morphology had no obvious influence on cell proliferation, which is consistent with others. $^{42}$

\section{Discussion}

To improve the efficacy of anti-TB drug in deeply skeletal system, a porous drug release delivery system has been attempted to encapsulate hydrophobic RIF into PCL MS by $\mathrm{o} / \mathrm{o}$ emulsion solvent evaporation method. The formation mechanism of the porous structure is depicted in Figure 5. In the initial stage, many small bubbles and droplets caused by air or entrapped fluid were contained in a big emulsion droplet in order to provide greater resistance to mechanical breakdown during the stirring. ${ }^{44}$ Then, the organic solvent gradually evaporated accompanied with polymer molecular chain shrinkage and precipitation. As the solvent evaporation started from the exterior of emulsion droplet, the shells of MS were formed first as previously reported. ${ }^{45}$ However, owing to the rapid solvent evaporation rate, the polymer precipitation was quite fast, resulting in partial solidification of the droplet and extensive shrinkage of polymer chain. At the same time, the small bubbles and droplets tended to move to the outside of MS. Thus, when they moved to the section where the polymer coagulated, the micropores formed and set in situ. Additionally, some bubbles or droplets had merged inside because of the smaller space and higher pressure, leading to bigger pores and empty spaces formed in the center. On the other hand, the porous shell structure also accelerated the removal rate of acetone, resulting in faster polymer solidification. Finally, after a thorough evaporation of acetone, the polymer shrinked and small particles with porous structures were fabricated.

Different RIF dosages also affect the porous structure formation of PMS. The micromorphology changes of PMS can be ascribed to the relatively high viscosity of emulsion drops caused by addition of more drugs. The influence of high viscosity on the extent of polymer chain as well as the movement of entrapped bubbles and droplets may baffle them from moving toward the surface of MS. Besides, it also affects the MS' size of PMS larger than that of DMS, because it is difficult to provide enough energy to disperse the high-viscosity oil phase as small as the low-viscosity one under the same stirring rate. Meanwhile, neither RIF nor PCL is soluble in liquid paraffin and, thus, the diffusion of RIF into the external oil phase is limited. As a result, the MS' encapsulation efficiency significantly increased. The diminishing entrapment efficiency demonstrates that

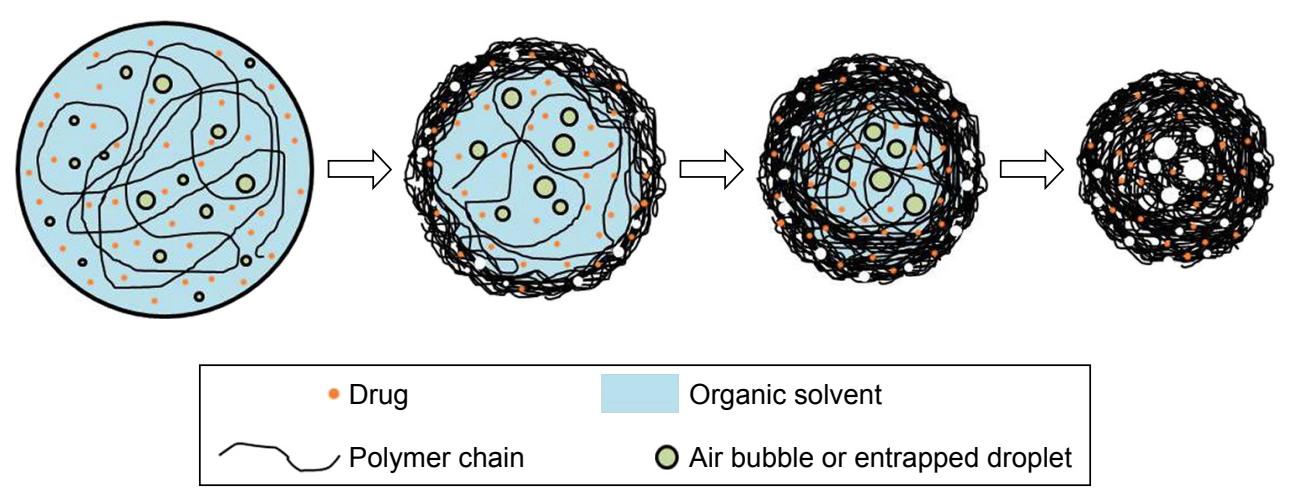

Figure 5 Hypothesis about the mechanism of formation of the porous microspheres by oil-in-oil (o/o) emulsion solvent evaporation method. 
more RIF diffused into the continuous phase. Furthermore, the optical micrograph of drug distribution corresponds to the theory that the hydrophobic drugs have higher affinity with PCL,${ }^{46}$ so that RIF distributed more evenly within PCL MS matrix.

In vitro release study indicates that RIF-loaded porous PCL MS could provide a longer and more sustained release compared with pure drug. However, the decrease in the cumulative release amount of RIF-loaded PMS suggests that the drug release profile is not only controlled by typical drug diffusion model. Therefore, it is necessary to investigate the RIF release kinetic mechanisms from the porous PCL MS. So far, numerous mathematical models have been developed to describe the drug release behavior of the controlledrelease system. ${ }^{47}$ To simplify the release property analysis, a well-known empirical exponential expression is widely introduced to describe the release behavior of drug release systems with different mechanisms and various geometries in many studies. ${ }^{30}$ According to the references, the kinetics of drug release indicates Fickian diffusion, zero-order release, and non-Fickian diffusion when $n$ is $0.43,1$, and $0.43-1$, respectively. ${ }^{48}$ Here, all data of the release exponent of PMS ranged from 0.53 to 0.73 , which was non-Fickian diffusion. It is quite different from the normal Fickian diffusion in nonporous PCL MS' release kinetics. ${ }^{49}$ Non-Fickian diffusion means that the drug released from the porous matrix is controlled by both diffusion and dissolution. ${ }^{50}$ In other words, the non-Fickian diffusion of RIF is mainly caused by two competitive factors: drug-loading content and porous structure of MS. When the release media penetrated into the MS through micropores, the initial RIF content was higher than its solubility in the microenvironment, leading to a diffusion-controlled release for RIF-loaded PMS. Taking into account the drug-loading content, a faster release rate is expected for the sample with a higher RIF-loading content. At the same time, in the view of the porous structures, more pores lead to the release media penetrating fully, and thus more drugs could dissolve from the matrix. When all of these factors are considered together, the MS with higher drugloading content and fewer pores on the surface presented a harmoniously controlled release for the RIF-loaded PMS.

It has been reported that RIF exhibits the minimal inhibitory concentration of $0.016 \mu \mathrm{g} / \mathrm{mL}$ for $S$. aureus. ${ }^{51} \mathrm{In}$ this study, RIF released from PMS maintained $>1 \mu \mathrm{g} / \mathrm{mL}$ throughout the release process in Figure 2. It is noteworthy to mention that the drug-loading rate and encapsulation efficiency of porous MS prepared by o/o method were much higher than the dense particles prepared in $\mathrm{o} / \mathrm{o}$ system (Table 1). Thus, an effective dose of RIF released from PMS is sufficient for the therapeutic target of the delivery system. The antibacterial results also demonstrate that the drug efficacy of RIF from PMS exhibited strong antibacterial activity against $S$. aureus.

Many researchers have adopted enzymatic degradation to evaluate polymers' degradation behavior. ${ }^{52,53}$ Although a number of lipases were utilized in degradation researches, lipase from Pseudomonas has been widely used to catalyze the PCL degradation because of its high hydrolyzing activity. It is well known that the enzymatic degradation occurred including two processes: 1) The enzyme was adsorbed on the surface of materials; 2) the materials started to degrade under the catalysis of enzyme, ${ }^{54}$ and presented a prior degradation at the amorphous regions of the particles. ${ }^{40}$ As observed in Figure 3, the difference in surface morphologies can be understood that the porous surface of PMS constitutes a larger area to adsorb enzyme than the smooth surface of DMS. As a result, the surface of porous MS changes significantly relative to that of DMS, which also support the view that the enzymatic degradation of PCL is a surface erosion process. ${ }^{54}$ However, based on the overall degradation rate test and crystallinity study as shown in Figure 3, the polymer crystallinity took the decisive influences on the degradation rate of these MS. This result is in accordance with D.R. Chen, ${ }^{55}$ who also revealed that surface area had no great influence on the degradation rate of the PCL sample. Therefore, the porous PCL MS still maintain the slow degradation property of PCL materials, which could avoid producing excess carboxylic acid end groups during the degradation and further affecting the stability of incorporated drug. ${ }^{56}$ From in vitro experiment with cells in Figure 4, the drug-loaded MS exhibit well cell adhesion and proliferation, which should be preferentially chosen for future in vivo investigation and application.

\section{Conclusion}

In this study, RIF-loaded PCL MS with porous structure and high encapsulation were successfully prepared by o/o emulsion solvent evaporation technique. The features of the MS, according to drug concentration, were investigated. Our results demonstrated that it was an efficient way to entrap slightly water-soluble drug like RIF into PCL by o/o emulsion solvent evaporation method with uniform drug distribution. The formation mechanism of the porous structure was postulated for drug diffusion investigation. Further, the drug release kinetic mechanism of PCL MS was changed by porous structures to non-Fickian diffusion, which is different from the dense one, and the RIF-loaded 
porous PCL MS could provide a prolonged and sustainedrelease behavior with an effective drug concentration against $S$. aureus. Meanwhile, the enzymatic degradation studies demonstrated that the polymer crystallinity had prominent influences on the degradation rate of MS regardless of the porous morphology, and, therefore, it could generate gentle environment by PCL slow degradation. Contemplating the above properties, it is promising that the RIF-loaded porous MS have the potential as a novel antibacterial drug delivery system used in skeletal treatment.

\section{Acknowledgments}

This work is supported by National 863 Project (No. 2015AA033702) and the China NSFC project (No. 31670965).

\section{Disclosure}

The authors report no conflicts of interest in this work.

\section{References}

1. Aristoff PA, Garcia GA, Kirchhoff PD, Showalter HD. Rifamycinsobstacles and opportunities. Tuberculosis (Edinb). 2010;90(2):94-118.

2. Tian WW, Wang QQ, Liu WD, Shen JP, Wang HS. Mycobacterium marinum: a potential immunotherapy for Mycobacterium tuberculosis infection. Drug Des Devel Ther. 2013;7:669-680.

3. Suarez-Garcia I, Noguerado A. Drug treatment of multidrug-resistant osteoarticular tuberculosis: a systematic literature review. Int $J$ Infect Dis. 2012;16(11):e774-e778.

4. Sato MR, Oshiro Junior JA, Machado RT, et al. Nanostructured lipid carriers for incorporation of copper(II) complexes to be used against Mycobacterium tuberculosis. Drug Des Devel Ther. 2017;11:909-921.

5. Companion Handbook to the WHO Guidelines for the Programmatic Management of Drug-Resistant Tuberculosis. Geneva: World Health Organization; 2014.

6. Ohashi K, Kabasawa T, Ozeki T, Okada H. One-step preparation of rifampicin/poly(lactic-co-glycolic acid) nanoparticle-containing mannitol microspheres using a four-fluid nozzle spray drier for inhalation therapy of tuberculosis. J Control Release. 2009;135(1):19-24.

7. Ruckh TT, Oldinski RA, Carroll DA, Mikhova K, Bryers JD, Popat KC. Antimicrobial effects of nanofiber poly(caprolactone) tissue scaffolds releasing rifampicin. J Mater Sci Mater Med. 2012;23(6):1411-1420.

8. Hong X, Wei L, Wu F, et al. Dissolving and biodegradable microneedle technologies for transdermal sustained delivery of drug and vaccine. Drug Des Devel Ther. 2013;7:945-952.

9. Dorniani D, Hussein MZ, Kura AU, Fakurazi S, Shaari AH, Ahmad Z. Preparation and characterization of 6-mercaptopurine-coated magnetite nanoparticles as a drug delivery system. Drug Des Devel Ther. 2013;7: 1015-1026.

10. Hirota K, Hasegawa T, Nakajima T, et al. Delivery of rifampicin-PLGA microspheres into alveolar macrophages is promising for treatment of tuberculosis. J Control Release. 2010;142(3):339-346.

11. Pandit S, Martin C, Alpar HO. Positively charged rifampicin-loaded microspheres for lung delivery. J Drug Deliv Sci Technol. 2005;15(4): 281-287.

12. Böstman OM, Pihlajamäki HK. Adverse tissue reactions to bioabsorbable fixation devices. Clin Orthop. 2000;371:216-227.

13. Barber FA, Spenciner DB, Bhattacharyya S, Miller LE. Biocomposite implants composed of Poly(Lactide-co-Glycolide) $/ \beta$-Tricalcium phosphate: systematic review of imaging, complication, and performance outcomes. Arthroscopy. 2017;33(3):683-689.
14. Chung HJ, Park TG. Surface engineered and drug releasing prefabricated scaffolds for tissue engineering. Adv Drug Deliv Rev. 2007; 59(4-5):249-262.

15. Hassan AH, Hosny KM, Murshid ZA, Alhadlaq A, Alyamani A, Naguib G. Depot injectable biodegradable nanoparticles loaded with recombinant human bone morphogenetic protein-2: preparation, characterization, and in vivo evaluation. Drug Des Devel Ther. 2015; 9(9):3599-3606.

16. Jung JH, Ree M, Kim H. Acid-and base-catalyzed hydrolyses of aliphatic polycarbonates and polyesters. Catalysis Today. 2006; 115(1-4):283-287.

17. Gleadall A, Pan J, Kruft MA, Kellomaki M. Degradation mechanisms of bioresorbable polyesters. Part 1. Effects of random scission, end scission and autocatalysis. Acta Biomater. 2014;10(5):2223-2232.

18. Higgins M, Watchorn D, Mcdonald C, Keane J, Mclaughlin AM. Therapeutic drug monitoring of isoniazid and rifampicin in acute TB treatment. Ir J Med Sci. 2014;183(1311):S538-S538.

19. Celikkaya E, Denkbaş EB, Pişkin E. Rifampicin carrying poly (D,Llactide)/poly(ethylene glycol) microspheres: loading and release. Artif Organs. 1996;20(7):743-751.

20. Wang X, Wang Y, Wei K, Zhao N, Zhang S, Chen J. Drug distribution within poly( $\varepsilon$-caprolactone) microspheres and in vitro release. $J$ Mater Process Technol. 2009;209(1):348-354.

21. Yan L, Jiang DM, Cao ZD, et al. Treatment of Staphylococcus aureusinduced chronic osteomyelitis with bone-like hydroxyapatite/poly amino acid loaded with rifapentine microspheres. Drug Des Devel Ther. 2015;9:3665-3676.

22. Dash TK, Konkimalla VB. Poly- $\varepsilon$-caprolactone based formulations for drug delivery and tissue engineering: a review. J Control Release. 2012;158(1):15-33.

23. Natarajan V, Krithica N, Madhan B, Sehgal PK. Formulation and evaluation of quercetin polycaprolactone microspheres for the treatment of rheumatoid arthritis. J Pharm Sci. 2011;100(1):195-205.

24. Mana Z, Pellequer Y, Lamprecht A. Oil-in-oil microencapsulation technique with an external perfluorohexane phase. Int J Pharm. 2007; 338(1-2):231-237.

25. Luciani A, Coccoli V, Orsi S, Ambrosio L, Netti PA. PCL microspheres based functional scaffolds by bottom-up approach with predefined microstructural properties and release profiles. Biomaterials. 2008; 29(36):4800-4807.

26. Wang S, Guo S, Cheng L. Disodium norcantharidate loaded poly(epsilon-caprolactone) microspheres I. Preparation and evaluation. Int J Pharm. 2008;350(1-2):130-137.

27. Tamaru S, Igura N, Shimoda M. Effectiveness of water-air and octanolair partition coefficients to predict lipophilic flavor release behavior from O/W emulsions. Food Chem. 2018;239:712-717.

28. Schröder A, Berton-Carabin C, Venema P, Cornacchia L. Interfacial properties and physical stability of $\mathrm{O} / \mathrm{W}$ emulsions containing hydrolyzed whey proteins. Food Hydrocolloids. 2017;73:129-140.

29. Luo PP, Li JD, Zuo Y, Wu X, Li YB. A Novel Antimicrobial NanoComposite Porous Material for Bone Repair. Mater Sci Forum. 2011; 695:517-520.

30. Ritger PL, Peppas NA. A simple equation for description of solute release I. Fickian and non-Fickian release from non-swellable devices in the form of slabs, spheres, cylinders or discs. J Control Release. 1987; $5(1): 23-36$.

31. Reinbold J, Hierlemann T, Hinkel H, et al. Development and in vitro characterization of poly(lactide-co-glycolide) microspheres loaded with an antibacterial natural drug for the treatment of long-term bacterial infections. Drug Des Devel Ther. 2016;10:2823-2832.

32. Qiao Y, Zhai Z, Chen L, Liu H. Cytocompatible 3D chitosan/ hydroxyapatite composites endowed with antibacterial properties: toward a self-sterilized bone tissue engineering scaffold. Chin Sci Bull. 2015;60(13):1193-1202.

33. Zhang Y, Sun L, Jiang J, Zhang X, Ding W, Gan Z. Biodegradationinduced surface change of polymer microspheres and its influence on cell growth. Polym Degrad Stab. 2010;95(8):1356-1364. 
34. Peng QY, Kang F, Li J, Yang XL. Degradation of a fullerene endcapped polycaprolactone by lipase AK. Chin Sci Bull. 2010;55(29): 3279-3282.

35. Ludueña LN, Alvarez VA, Vazquez A. Processing and microstructure of PCL/clay nanocomposites. Mater Sci Eng. 2007;460-461(460): 121-129.

36. Li K, Zuo Y, Zou Q, et al. Synthesis and characterization of injectable nano-hydroxyapatite/polyurethane composite cement effective formulations for management of osteoporosis. J Nanosci Nanotechnol. 2016;16(12):12407-12417.

37. Lin JH, Chen CK, Wen SP, Lou CW. Poly-L-lactide/sodium alginate/ chitosan microsphere hybrid scaffolds made with braiding manufacture and adhesion technique: solution to the incongruence between porosity and compressive strength. Mater Sci Eng C Mater Biol Appl. 2015;52: 111-120.

38. Lakhkar NJ, Park JH, Mordan NJ, et al. Titanium phosphate glass microspheres for bone tissue engineering. Acta Biomater. 2012;8(11): 4181-4190.

39. Miao ZM, Cheng SX, Zhang XZ, Wang QR, Zhuo RX. Degradation and drug release property of star poly ( $\varepsilon$-caprolactone) $\mathrm{s}$ with dendritic cores. J Biomed Mater Res B Appl Biomater. 2007;81B(1):40-49.

40. Jenkins M, Harrison K. The effect of crystalline morphology on the degradation of polycaprolactone in a solution of phosphate buffer and lipase. Polym Adv Technol. 2008;19(12):1901-1906.

41. Li X, Liu H, Wang J, Li C. Preparation and characterization of poly( $\varepsilon$ caprolactone) nonwoven mats via melt electrospinning. Polymer. 2012; 53(1):248-253.

42. Kulkarni A, Reiche J, Hartmann J, Kratz K, Lendlein A. Selective enzymatic degradation of poly(epsilon-caprolactone) containing multiblock copolymers. Eur J Pharm Biopharm. 2008;68(1):46-56.

43. Durán N, Alvarenga MA, Da SE, Melo PS, Marcato PD. Microencapsulation of antibiotic rifampicin in poly(3-hydroxybutyrate-co-3hydroxyvalerate). Arch Pharmacal Res. 2008;31(11):1509-1516.

44. Xu Q, Czernuszka JT. Controlled release of amoxicillin from hydroxyapatite-coated poly(lactic-co-glycolic acid) microspheres. J Control Release. 2008;127(2):146-153.
45. Cowsar DR, Tice TR, Gilley RM, English JP. Poly (lactide-co-glycolide) microcapsules for controlled release of steroids. Methods Enzymol. 1985;112(4):101-116.

46. Wang X, Wang Y, Wei K, Zhao N, Zhang S, Chen J. Drug distribution within poly ( $\varepsilon$-caprolactone) microspheres and in vitro release. $J$ Mat Proc Technol. 2009;209(1):348-354.

47. Khan MA. Shafeeq T. Role of mathematical modeling in controlled drug delivery. J Sci Res. 2009;1(3):539-550.

48. Wang S, Guo S, Cheng L. Disodium norcantharidate loaded poly ( $\varepsilon$-caprolactone) microspheres: I. Preparation and evaluation. Int $J$ Pharm. 2008;350(1):130-137.

49. Yu L, Zhang H, Cheng SX, Zhuo RX, Li H. Study on the drug release property of cholesteryl end-functionalized poly ( $\varepsilon$-caprolactone) microspheres. J Biomed Mater Res Part B: Appl Biomater. 2006;77(1): $39-46$.

50. Chandrasekaran S, Paul D. Dissolution-Controlled transport from dispersed matrixes. J Pharm Sci. 1982;71(12):1399-1402.

51. Coe CJ, Doss SA, Tillotson GS, Amyes SG. Interaction of sub-inhibitory concentrations of ciprofloxacin and rifampicin against Staphylococcus aureus. Int J Antimicrob Agents. 1995;5(2):135-139.

52. Calil M, Gaboardi F, Bardi M, Rezende M, Rosa D. Enzymatic degradation of poly ( $\varepsilon$-caprolactone) and cellulose acetate blends by lipase and $\alpha$-amylase. Polym Test. 2007;26(2):257-261.

53. Liu L, Li S, Garreau H, Vert M. Selective enzymatic degradations of poly (L-lactide) and poly( $\varepsilon$-caprolactone) blend films. Biomacromolecules. 2000;1(3):350-359.

54. Herzog K, Muller RJ, Deckwer WD. Mechanism and kinetics of the enzymatic hydrolysis of polyester nanoparticles by lipases. Polym Degrad Stab. 2006;91(10):2486-2498.

55. Chen DR, Bei JZ, Wang SG. Polycaprolactone microparticles and their biodegradation. Polym Degrad Stab. 2000;67(3):455-459.

56. Ramesh DV. Comparison of oil-in-oil, water-in-oil-in-water and melt encapsulation techniques for the preparation of controlled release B12 poly(e-caprolactone) microparticles. Trends Biomater ArtifOrgans. 2010; 23(1):117-120.
Drug Design, Development and Therapy

\section{Publish your work in this journal}

Drug Design, Development and Therapy is an international, peerreviewed open-access journal that spans the spectrum of drug design and development through to clinical applications. Clinical outcomes, patient safety, and programs for the development and effective, safe, and sustained use of medicines are the features of the journal, which

\section{Dovepress}

has also been accepted for indexing on PubMed Central. The manuscript management system is completely online and includes a very quick and fair peer-review system, which is all easy to use. Visit http://www.dovepress.com/testimonials.php to read real quotes from published authors. 